IMECE2013-63829

\title{
A BIOMECHANICAL STUDY OF DIRECTIONAL MECHANICAL PROPERTIES OF PORCINE SKIN TISSUES
}

\author{
Hsiao-Ying Shadow Huang* \\ North Carolina State University \\ Department of Mechanical and Aerospace \\ Engineering \\ Raleigh, NC, USA \\ hshuang@ncsu.edu
}

Peter M. Prim

North Carolina State University

Fitts Department of Industrial and Systems Engineering

Raleigh, NC, USA

pmprim@ncsu.edu

\author{
Siyao Huang \\ North Carolina State University \\ Department of Mechanical and \\ Aerospace Engineering \\ Raleigh, NC, USA \\ shuang4@ncsu.edu
}

\author{
Taylor Gettys \\ North Carolina State University \\ Department of Mechanical and Aerospace \\ Engineering \\ Raleigh, NC, USA \\ tagettys@ncsu.edu \\ Ola L. Harrysson \\ North Carolina State University \\ Raleigh, NC, USA \\ harrysson@,ncsu.edu
}

Fitts Department of Industrial and Systems Engineering

\begin{abstract}
Skin is a multilayered composite material and composed principally of the proteins collagen, elastic fibers, and fibroblasts. The direction-dependent material properties of skin tissue is important for physiological functions like skin expansion. The current study has developed methods to characterize the directional biomechanical properties of porcine skin tissues. It is observed that skin tissue has a nonlinear anisotropy biomechanical behavior, where the parameters of material stiffness is $378 \pm 160 \mathrm{kPa}$ in the preferred-fiber direction and $65.96 \pm 40.49 \mathrm{kPa}$ in the cross-fiber direction when stretching above $30 \%$ strain equibiaxially. The results from the current study will help optimize functional skin stretching for patients requiring large surface area skin grafts and reconstructions due to burns or other injuries.
\end{abstract}

\section{INTRODUCTION}

Skins are complex in their structure and composition, and the properties of the skin vary from species to species [1-4]. It is comprised of several different types of proteins (e.g., collagens, elastin and proteoglycans) aligned in different directions and have molecular chains that have a directional orientation $[5,6]$. A previous study reported various mechanical properties before and after skin stretching, though failed to identify preferred-fiber and cross-fiber directions before mechanical characterizations [7].

Mechanical testing under uniaxial tensile loading allows lateral contraction of the specimen during extension, which can further facilitate axial elongation [7]. Biaxial testing does not permit this process (or, more specifically, does so to a programmable degree), and hence skin loaded biaxially will facilitate accurate delineation of mechanical properties. Fibers composed of types I, III, and V collagens are found in the tissue [8] and studies have shown that the spaces between collagen bundles change, reflecting a variation in collagen fiber directions $[9,10]$.

In regenerative medicine, a significant effort is focused on increasing the area and volume of skin tissue using mechanical strain methods. The main intent is to plastically deform this biological material so that its surface area increases and over time regain its full thickness due to cell proliferation in a bioreactor. The driving force is the high fatality of severely burned patients with a large area of thermally or chemically burned skin tissue. To treat patients with severe burns, small skin grafts can be harvested from healthy places on the body, quickly expanded to much larger areas in vitro, compared to the original area, and then be transplanted back to a burnt area of the body. Thus, skin expansion has become a valuable method in plastic and reconstructive surgery [11] [12]. However, current prototype skin expansion bioreactors have been reported to cause tissue tearing due to uneven loading, gripping methods, stress concentration, and other factors intrinsic to the tissue properties [5]. Tissue tearing is the key obstacle faced by in-vitro bioreactors, hindering many potential medical applications, such as reconstructing birth defects, burn injuries, or breast tissue after mastectomy. Therefore, the current study aims to provide a method to better obtain fundamental quantitative data delineating direction-dependent mechanical properties of porcine skin tissue. It is expected that the identification of directional mechanical properties will optimize functional surface area enlargement of skin tissue, and hence better serve burned patients in health-care communities. 


\section{MATERIALS AND METHOD}

\section{Sample preparation}

Briefly, porcine skins from the belly area of a 6-month-old pig were obtained from the Swine Education Unit at North Carolina State University immediately after euthanization for reasons unrelated to the current project, and tissues were prepared with a dermatome. Tissue samples were returned to the laboratory within 60 minutes after being euthanized. Subcutaneous fat tissue was removed and samples were then stretched out and held taut while being cut (Fig. 1(a)).

A total of six tissue samples $(\sim 7 \mathrm{~mm} \times 7 \mathrm{~mm})$ were prepared (Fig. 1(b)). A micrometer was used to measure the thickness of the samples and the samples were stored in Hank's Balance Salt Solution (HBSS) for the relaxation.

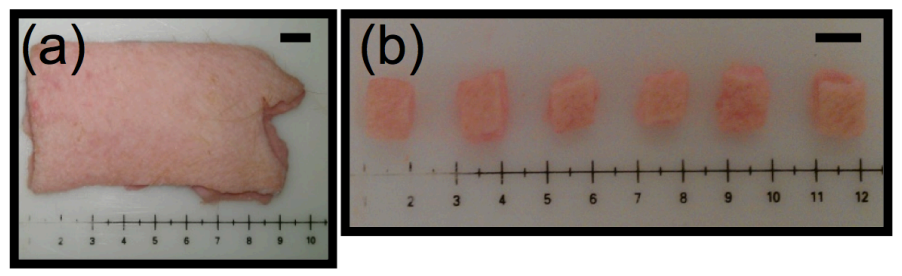

Figure 1: Sample preparation. (a) Tissue samples were returned to the laboratory within 60 minutes after being euthanized. Subcutaneous fat tissue was removed and samples were then stretched out and held taut while being cut. (b) A total of six tissue samples $(\sim 7 \mathrm{~mm} \times 7 \mathrm{~mm})$ were prepared. Scale bar $=1 \mathrm{~mm}$.

\section{Mechanical characterization of skin tissue}

Though biaxial testing of skin has been studied by Lanir and Fung [13-15], the current work focuses on establishing a method to characterize direction-dependent mechanical properties. A biaxial tissue tester (BioTester 5000, CellScale, Waterloo, Canada), equipped with two load cells ( $500 \mathrm{mN} \pm 1$ $\mathrm{mN}$ ) and actuators for each axis of loading, was used for measuring the force and displacement of the skin tissues [16] (Fig. 2(a)). The measured values were used to further obtain stress-strain curves and to calculate the parameters of material's stiffness [17-19]. Synchronized time lapse video for real-time monitoring and post-process analysis was provided by the charged-couple device (CCD) camera, which acquires images with a pixel resolution of $1280 \times 960$ at an acquisition rate of 15 $\mathrm{Hz}$, with a lens focal length of $75 \mathrm{~mm}$. A temperature controlled saline bath with data logging capability provided a physiological environment for testing soft tissue specimens.

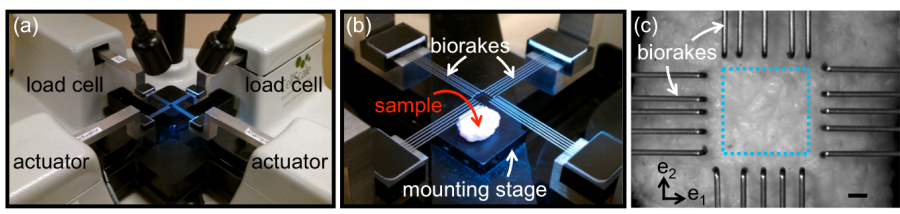

Figure 2: The Biotester is capable of applying physiological plausible biaxial loading and stretching states to tissue samples. (a) The biotester is equipped with two load cells and two actuators for each axis. (b) The mounting stage enables samples being pierced through by the tungsten biorakes. (c) The biorakes provide evenly distributed loading/stretching states and this unique feature assures the control of loading conditions. Scale $\mathrm{bar}=1 \mathrm{~mm}$.
Specimen mounting is considered as one of the major challenges of biaxial testing [16]. For example, artifacts such as suturing procedures usually cause discrepancy in results due to inconsistent boundary conditions $[16,20]$. In contrast, biorakes provide fast and accurate sample mounting: each biorake consists of five tungsten tines used to anchor one edge of the specimen [16-19, 21] (Fig. 2b). Four rakes provide uniform attachment across the edges of the samples and evenly distribute the load spanning $4 \mathrm{~mm}$ in length on each side of the sample (Fig. 2c). This unique feature not only assures the control of boundary conditions, but also significantly reduces the variability between sample sizes. In other words, if the sample size prepared is larger than $4 \mathrm{~mm}^{2}$, the active loading area remains 4-mm $\times 4-\mathrm{mm}$. After the sample was mounted, the sample was lowered into the HBSS bath, which was heated to $37^{\circ} \mathrm{C}$ to simulate an in vivo physiological environment.

The tissue samples were tested up to $35 \%$ strain in both axes with a 15 second stretch cycle and a 15 second recovery cycle, with no hold time (Fig. 3(a)). After the sample was mounted, one test was executed to pre-stretch the tissue to release the residual stresses inside tissue samples, i.e., pre-conditioning. The result of the pre-conditioning was not included in the material property measurements. Each sample was then tested five times, as defined as one set of tests. The image tracking and analysis software (LabJoy, CellScale, Waterloo, Canada), an integrated analysis module for Biotester, was used to output corresponding force data and to generate force vs. displacement relationships for both directions in real time during the equibiaxial testing (Fig. 3(b)(c)).
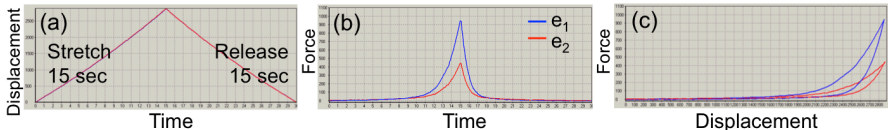

Figure 3: (a) The tissue samples are tested up to $35 \%$ strain in both axes with a 15 second stretch cycle and a 15 second recovery cycle, with no hold time. (b) An integrated analysis module for Biotester outputs measured force data in both directions. (c) The module also generates force vs. displacement relationships for both directions in real time during the equibiaxial testing.

\section{Collagen fiber directions identification}

When tissue samples are prepared, it is difficult to visualize preferred and cross-preferred collagen fiber directions. The minimized coupling effects between preferred and cross-fiber directions are generally observed when their mechanical properties exhibit the most differences between them, i.e., anisotropy. Therefore, to identify preferred and cross-fiber directions, the samples are rotated counterclockwise at approximately 25-30 degrees after each set of tests. Due to the symmetry, three testing angles are used: 0-degree, 30-degree and 60-degree. In Figure 4, 0-degree samples ' $a$ ' were prepared as described previously and as shown in Figure 2(c), in which sample edges were aligned with stretching directions, $\mathrm{e}_{1}$ and $\mathrm{e}_{2}$ directions respectively. Samples ' $\mathrm{b}$ ' were the ones after 30-degree rotation from the sample edges, and samples 'c' were the ones with a total of 60-degree rotation from the sample edges (Fig. 4). The method is developed to identify the preferred- and cross-fiber directions of the tissue and to 
determine the intrinsic direction-dependent mechanical property of skin samples (Fig. 4).

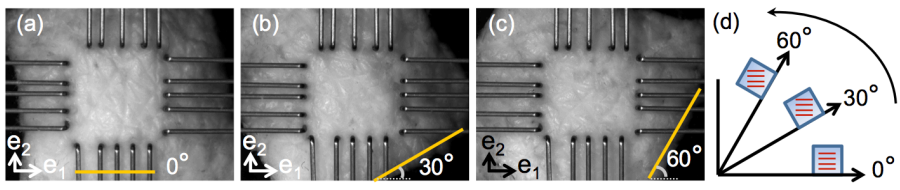

Figure 4: The samples are rotated counterclockwise at approximately 30 degrees after each test to determine the direction-dependent mechanical property of skin samples. $\mathrm{e}_{1}$ and $\mathrm{e}_{2}$ directions are defined as stretching directions. Preferred-collagen fibers orientation is represented in color red, as shown in (d).

\section{Statistical analysis}

Data is presented as the mean \pm standard deviation. The number of experimental samples is represented as $n$. Student's $\mathrm{t}$ tests are used to test differences in population means. Differences with $p<0.05$ are considered significant.

\section{RESULT AND DISCUSSION}

The displacements are calculated based on the relative position of the biorakes, and the force data is directly output from the load cells. After averaging over measured stress vs. strain for tissue samples $(\mathrm{n}=6)$ under equibiaxial testing, the results are shown in Figure 5. The plot shows the correlation between the strain and the resulting stress in the preferred-fiber and cross-fiber directions for skin tissue samples. In Figure 5, clear non-linear and anisotropy material behaviors (solid vs. dashed lines) of the skin tissue are observed in all samples at different testing angles. In addition, three zones are observed: zone 1 is between $0 \%$ and $25 \%$ strain stretching, zone 2 is between $25 \%$ and $30 \%$ strain stretching, and zone 3 is between $30 \%$ and $35 \%$ strain stretching. Under the equal-biaxial stretching, it is observed that high standard deviations exist when samples are tested above $30 \%$ strain. It could be due to the sensitivity of the load cells and where forces data were collected.

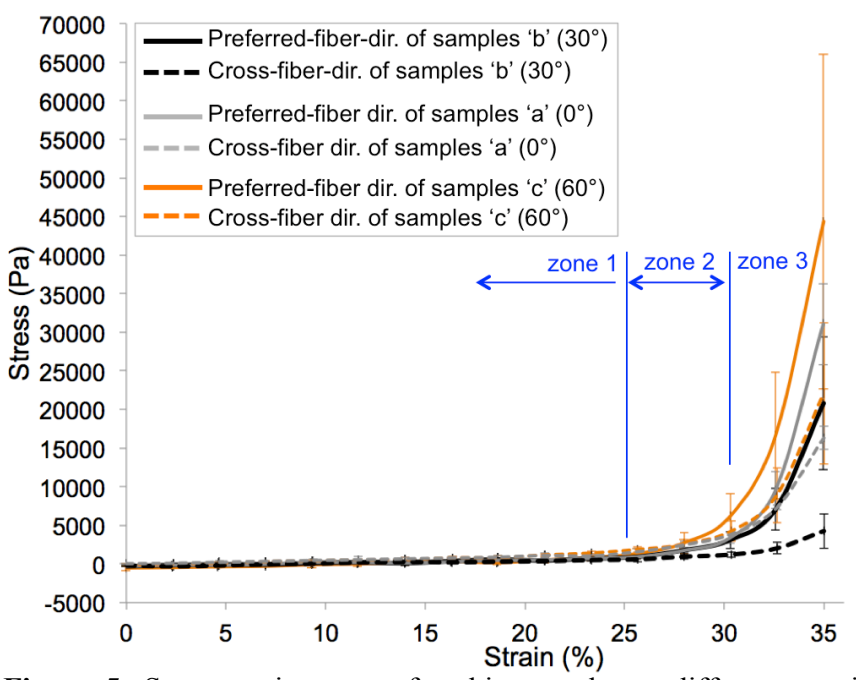

Figure 5: Stress-strain curves for skin samples at different rotation angles $(n=6)$. A nonlinear mechanical property is observed. Three zones are observed: zone 1 ( $0-25 \%$ strain), zone 2 (25-30\% strain), and zone 3 (30-35\% strain).
It is observed that samples at 30-degree testing angles exhibit the most differences in mechanical properties between preferred and cross-fiber directions (Fig. 5). It is due to the fact that most of the collagen fibers are straightened and aligned along the $\mathrm{e}_{1}$ stretching direction at this testing angle (Fig. 6b). Therefore, a more compliant material property is measured in the cross-stretching direction, $e_{2}$ (Fig. 5). In contrast, collagen fibers in samples at other testing angles are not perfectly aligned along and perpendicular to both stretching directions $\mathrm{e}_{1}$ and $\mathrm{e}_{2}$, respectively. Therefore, anisotropic behaviors of tissue samples are less obviously comparing to the ones at the 30-degree testing angles.

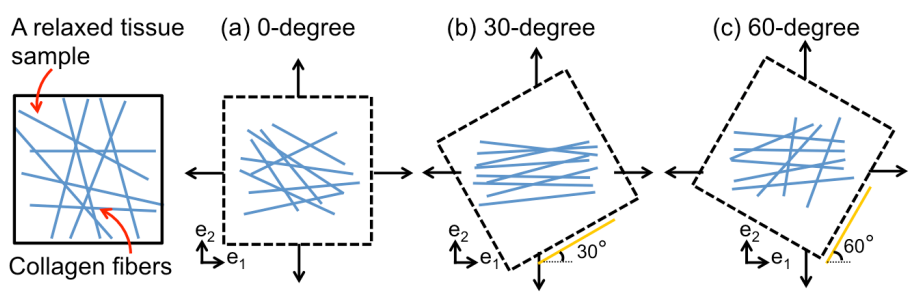

Figure 6: Collagen fibers are randomly distributed in a relaxed tissue sample. From the stress-strain curves in Figure 5, it is observed that samples at 30-degree testing angles have the most differences in mechanical properties between preferred and cross-fiber directions. It is implied that most of collagen fibers are straightened and aligned along the $\mathrm{e}_{1}$ stretching direction at this testing angle, as shown in (b). In contrast, collagen fibers in samples at other testing angles are not perfectly aligned along and perpendicular to both stretching directions $\mathrm{e}_{1}$ and $\mathrm{e}_{2}$, as shown in (a) and (c). Therefore, anisotropic behaviors of tissue samples are less obviously comparing to the ones at the 30degree testing angles, as shown in Figure 5.

Piecewise parameters of material stiffness of samples ' $b$ ' in three zones are listed in Table 1, and values are expressed as means \pm standard deviations for skin tissue samples. Stressstrain curves in zone 1 provide moduli of elasticity of samples ' $b$ ' and stress-strain curves in zones 2 and 3 provide tangent moduli of elasticity. The differences between the directiondependent stress vs. strain curves in samples ' $b$ ' are mainly due to collagen fibers arrangements [22-25]. Most of the collagen fibers in samples ' $b$ ' align along the $e_{1}$ direction, therefore stiffer mechanical properties in the preferred-fiber-direction are observed (Fig. 5 and Fig. 6b).

Table 1: Piecewise parameters of materials stiffness of samples ' $b$ ' at 30-degree testing angle.

\begin{tabular}{|c|c|c|c|}
\hline Modulus (kPa) & $\begin{array}{l}\mathbf{0 - 2 5 \%} \text { of } \\
\text { strain } \\
\text { (Zone 1) }\end{array}$ & $\begin{array}{l}\mathbf{2 5 - 3 0 \% \text { of }} \\
\text { strain } \\
\text { (Zone 2) }\end{array}$ & $\begin{array}{l}\mathbf{3 0 - 3 5 \% \text { of }} \\
\text { strain } \\
\text { (Zone 3) }\end{array}$ \\
\hline r-direction (p) & $4.66 \pm 1.01$ & $46.70 \pm 17.64$ & $378.01 \pm 160.13$ \\
\hline Cross-fiber-direction (c) & $3.05 \pm 0.65$ & $13.27 \pm 0.93$ & $65.96 \pm 40.49$ \\
\hline
\end{tabular}

In zone $1(0-25 \%$ strain), it is observed that the stiffness in the preferred-fiber-direction is higher than that in the crossfiber-direction $\left(\mathrm{E}_{\mathrm{p}}^{(1) /} \mathrm{E}_{\mathrm{c}}^{(1)}=1.53\right)$, suggesting that the skin sample has slightly anisotropic material properties in zone 1 (Table 1 and Fig. 5). This could be due to randomly distributed collagen fibers in skin tissue samples being not fully aligned along the preferred-collagen fiber direction before reaching $25 \%$ equibiaxial stretching, as shown in Figure 5. In zone 2 (25- 
$30 \%$ strain), it is observed that the stiffness in the preferredfiber-direction is more than 3 times higher than that in the crossfiber-direction $\left(E_{p}^{(2)} / E_{c}^{(2)}=3.52\right)$, as shown in Table 1 and Figure 5. It is suggested that the skin sample has anisotropic material properties in zone 2 (Table 1 and Fig. 5). However, it is still not clear which intrinsic biological characteristic gives rise to the interesting microstructure features of collagen fibers that reflect back to the measured mechanical property in zone 2 . In zone 3 (30-35\% strain), it is observed that the stiffness in the preferred-fiber-direction is more than five times higher than that in the cross-fiber-direction for the skin tissue samples $\left(\mathrm{E}_{\mathrm{p}}{ }^{(3)} / \mathrm{E}_{\mathrm{c}}{ }^{(3)}\right.$ = 5.73) (Table 1 and Fig. 5). The increased ratio of this mechanical property in the skin tissue samples suggests that collagen fibers experience most realignment and straightening in zone 3.

Comparing to the result in zone 1 , a ten-fold increase in the stiffness in the preferred-fiber-direction is observed $\left(\mathrm{E}_{\mathrm{p}}{ }^{(2)} / \mathrm{E}_{\mathrm{p}}{ }^{(1)}=\right.$ 10.02). In contrast, only a four-fold increase in the stiffness in the cross-fiber-direction is observed $\left(\mathrm{E}_{\mathrm{c}}{ }^{(2)} / \mathrm{E}_{\mathrm{c}}{ }^{(1)}=4.35\right)$. The result confirms that this highly anisotropic material property in skin tissues is dominated by the orientation of the collagen fibers [26-28]. Moreover, the stiffness of the skin tissue samples in the preferred-fiber-direction is eight-fold higher than the one in zone $2\left(\mathrm{E}_{\mathrm{p}}{ }^{(3)} / \mathrm{E}_{\mathrm{p}}{ }^{(2)}=8.09\right)$, which is slightly lower than the one in zone $1\left(\mathrm{E}_{\mathrm{p}}^{(2)} / \mathrm{E}_{\mathrm{p}}{ }^{(1)}=10.02\right)$. Interestingly, the stiffness of the skin tissue samples in the cross-fiber-direction is comparable to the one in zones 2 and $1\left(\mathrm{E}_{\mathrm{c}}^{(3)} / \mathrm{E}_{\mathrm{c}}{ }^{(2)}=4.97\right.$ and $\mathrm{E}_{\mathrm{c}}{ }^{(2)} / \mathrm{E}_{\mathrm{c}}{ }^{(1)}=$ 4.35). It is suggested that collagen fiber alignment in skin tissues is saturated when samples are stretched above $30 \%$ strain (Table 1 and Fig. 5). In the current in-vitro study, it was recognized in pilot experiments that skin samples began to tear when stretched above $35 \%$ strain, consistent with observations reported in other studies [7]. In this study, it is intended to conduct a parametric study to understand direction-dependent mechanical properties. Therefore, tissue samples are equibiaxially stretched only up to $35 \%$ strain.

Hysteresis is observed during equibiaxial testing (Fig. 3c), therefore viscoelasticity play an important role in the mechanical behavior of skin tissue. Viscoelasticity of skin tissues have been characterized previously [7, 29], and it is observed that the stress relaxation of the tissue is highly dependent on the duration of the tissue stretching. Moreover, the results from the studies also revealed that the skin tissue slowly recovers back to the original mechanical properties. However, the studies failed to identify preferred-fiber and cross-fiber directions prior to these mechanical measurements. Therefore, the current study aims to establish a measurement method to identify and quantify direction-dependent mechanical properties of skin tissue prior to viscoelasticity measurement. The follow up study focusing on directionalviscoelasticity of skin tissue prior to and after skin stretching is currently in preparation. Moreover, surface-area gain for reconstructive surgery has been studied by varying tissue expander shapes [30] and tissue expansion speed and duration [29], and has been characterized by histochemical [31], histomorphological and ultrastructural analyses [5]. Therefore, the demonstrated method in the current study provides a great foundation on optimizing surface-area gain for the reconstructive surgery. That is, by stretching skin tissue with proper direction, force, speed, and duration, it is expected to achieve a functional tissue expansion and helping patients who need a large surface area of skin grafts or reconstructions.

\section{CONCLUSION}

Biological tissues have a complex microstructure and their biomechanical behaviors are highly related to inhomogeneous collagen fiber architecture. The current study provides a method to quantify directional mechanical properties of skin tissues, which is very important prior to reporting mechanical properties of skin tissues. To the authors' knowledge, it is the first reported directional biomechanical properties of skin tissue. The directional-mechanical properties of skin tissue is measured biaxially and identified via the anisotropic behavior of tissue samples. It is observed that skin tissue has a nonlinear anisotropy biomechanical behavior, where the parameters of material stiffness is $378 \pm 160 \mathrm{kPa}$ in the preferred-fiber direction and $65.96 \pm 40.49 \mathrm{kPa}$ in the cross-fiber direction when stretching above $30 \%$ strain equibiaxially. The result from the current study provides a strong foundation for the skin stretching optimization, where the increased surface-area is required in modern skin grafts and reconstructions.

\section{REFERENCE}

[1] Silver, F. H., Siperko, L. M., and Seehra, G. P., 2003, "Mechanobiology of force transduction in dermal tissue," Skin Research and Technology, 9(1), pp. 3-23.

[2] Silver, F. H., Seehra, G. P., Freeman, J. W., and DeVore, D., 2002, "Viscoelastic properties of young and old human dermis: A proposed molecular mechanism for elastic energy storage in collagen and elastin," Journal of Applied Polymer Science, 86(8), pp. 1978-1985.

[3] Silver, F. H., Freeman, J. W., and DeVore, D., 2001, "Viscoelastic properties of human skin and processed dermis," Skin Research and Technology, 7(1), pp. 18-23.

[4] Silver, F. H., Kato, Y. P., Ohno, M., and Wasserman, A. J., 1992, "Analysis of Mammalian Connective-Tissue - Relationship between Hierarchical Structures and Mechanical-Properties," Journal of longterm effects of medical implants, 2(2-3), pp. 165-198.

[5] Ladd, M. R., Lee, S. J., Atala, A., and Yoo, J. J., 2009, "Bioreactor Maintained Living Skin Matrix," Tissue Engineering Part a, 15(4), pp. 861-868.

[6] Daly, C. H., 1982, "Biomechanical Properties of Dermis," Journal of Investigative Dermatology, 79, pp. S17-S20.

[7] Zeng, Y. J., Huang, K., Xu, C. Q., Zhang, J., and Sun, G. C., 2001, "Biorheological characteristics of skin after expansion," Biorheology, 38(5-6), pp. 367-378.

[8] Weber, L., Kirsch, E., Muller, P., and Krieg, T., 1984, "Collagen Type Distribution and Macromolecular Organization of ConnectiveTissue in Different Layers of Human-Skin," Journal of Investigative Dermatology, 82(2), pp. 156-160.

[9] Lavker, R. M., Zheng, P. S., and Dong, G., 1987, "Aged Skin - a Study by Light, Transmission Electron, and Scanning ElectronMicroscopy," Journal of Investigative Dermatology, 88(3), pp. S44S51.

[10] Mays, P. K., Bishop, J. E., and Laurent, G. J., 1988, "AgeRelated-Changes in the Proportion of Type-i and Type-Iii Collagen," 
Mechanisms of ageing and development, 45(3), pp. 203-212.

[11] Argenta, L. C., Watanabe, M. J., and Grabb, W. C., 1983, "The use of Tissue Expansion in Head and Neck Reconstruction," Annals of Plastic Surgery, 11(1), pp. 31-37.

[12] Arneja, J. S., and Gosain, A. K., 2007, "Giant Congenital Melanocytic Nevi," Plastic and Reconstructive Surgery, 120(2), pp. 26E-40E.

[13] Lanir, Y., and Fung, Y. C., 1974, "2-Dimensional MechanicalProperties of Rabbit Skin .1. Experimental System," Journal of Biomechanics, 7(1), pp. 29-34.

[14] Lanir, Y., and Fung, Y. C., 1974, "2-Dimensional MechanicalProperties of Rabbit Skin .2. Experimental Results," Journal of Biomechanics, 7(2), pp. 171-\&.

[15] Tong, P., and Fung, Y.-C., 1976, "The stress-strain relationship for the skin," Journal of Biomechanics, 9(10), p. 649.

[16] Eilaghi, A., Flanagan, J. G., Brodland, G. W., and Ethier, C. R., 2009, "Strain Uniformity in Biaxial Specimens is Highly Sensitive to Attachment Details," Journal of Biomechanical EngineeringTransactions of the Asme, 131(9), pp. 091003-091003.

[17] Huang, H.-Y. S., Balhouse, B. N., and Huang, S., "A Synergy Study of Heart Valve Tissue Mechanics, Microstructures, and Collagen Concentration," Proc. 2012 ASME International Mechanical Engineering Congress and Exposition, ASME.

[18] Huang, H.-Y. S., and Huang, S., "Real-Time Strain Mapping via Biaxial Stretching in Heart Valve Tissues," Proc. 2012 IEEE Engineering in Medicine and Biology Society, IEEE.

[19] Huang, H.-Y. S., Balhouse, B. N., and Huang, S., 2012, "Application of Simple Biomechanical and Biochemical Tests to Heart Valve Leaflets: Implications for Heart Valve Characterization and Tissue Engineering," Proceedings of the Institution of Mechanical Engineers, Part H: journal of Engineering in Medicine, 226(11), pp. 868-876.

[20] Sun, W., Sacks, M. S., and Scott, M. J., 2005, "Effects of boundry conditions on the estimation of the planar biaxial mechanical properties of soft tissues," Journal of Biomechanical EngineeringTransactions of the Asme, 127(4), pp. 709-715.

[21] Eilaghi, A., Flanagan, J. G., Tertinegg, I., Simmons, C. A., Brodland, G. W., and Ethier, C. R., 2010, "Biaxial mechanical testing of human sclera," Journal of Biomechanics, 43(9), pp. 1696-1701.

[22] Christie, G. W., and Barrattboyes, B. G., 1995, "MECHANICALPROPERTIES OF PORCINE PULMONARY VALVE LEAFLETS HOW DO THEY DIFFER FROM AORTIC LEAFLETS," Annals of thoracic surgery, 60(2), pp. S195-S199.

[23] Joyce, E. M., Liao, J., Schoen, F. J., Mayer, J. E., Jr., and Sacks, M. S., 2009, "Functional Collagen Fiber Architecture of the Pulmonary Heart Valve Cusp RID F-3703-2011," Annals of thoracic surgery, 87(4), pp. 1240-1249.

[24] Sacks, M. S., Smith, D. B., and Hiester, E. D., 1998, "The aortic valve microstructure: Effects of transvalvular pressure," Journal of Biomedical Materials Research, 41(1), pp. 131-141.

[25] Billiar, K. L., and Sacks, M. S., 2000, "Biaxial mechanical properties of the natural and glutaraldehyde treated aortic valve cusp Part I: Experimental results," Journal of Biomechanical EngineeringTransactions of the Asme, 122(1), pp. 23-30.

[26] Sacks, M. S., 2003, "Incorporation of experimentally-derived fiber orientation into a structural constitutive model for planar collagenous tissues," J Biomech Eng, 125(2), pp. 280-287.

[27] Sacks, M. S., and Sun, W., 2003, "Multiaxial mechanical behavior of biological materials," Annu Rev Biomed Eng, 5, pp. 251284.

[28] Sacks, M. S., 2000, "Biaxial mechanical evaluation of planar biological materials," Journal of Elasticity, 61, pp. 199-246.
[29] Zeng, Y. J., Xu, C. Q., Yang, J., Sun, G. C., and Xu, X. H., 2003, "Biomechanical comparison between conventional and rapid expansion of skin," British journal of plastic surgery, 56(7), pp. 660666.

[30] El Ghalbzouri, A., Lamme, E. N., van Blitterswijk, C., Koopman, J., and Ponec, M., 2004, "The use of PEGT/PBT as a dermal scaffold for skin tissue engineering," Biomaterials, 25(15), pp. 2987-2996.

[31] Wollina, U., Berger, U., Stolle, C., Stolle, H., Schubert, H., Zieger, M., Hipler, C., and Schumann, D., 1992, "Tissue Expansion in Pig Skin - a Histochemical Approach," Anatomia Histologia Embryologia-Journal of Veterinary Medicine Series C-Zentralblatt Fur Veterinarmedizin Reihe C, 21(2), pp. 101-111. 